This is an electronic reprint of the original article. This reprint may differ from the original in pagination and typographic detail.

Author(s): Nozaki, D.; Gomes da Rocha, Claudia; Pastawski, H.M.; Cuniberti, G.

Title: Disorder and dephasing effects on electron transport through conjugated molecular wires in molecular junctions

Year: $\quad 2012$

Version:

Please cite the original version:

Nozaki, D., Gomes da Rocha, C., Pastawski, H.M., \& Cuniberti, G. (2012). Disorder and dephasing effects on electron transport through conjugated molecular wires in molecular junctions. Physical Review B, 85, 155327.

https://doi.org/10.1103/PhysRevB.85.155327

All material supplied via JYX is protected by copyright and other intellectual property rights, and duplication or sale of all or part of any of the repository collections is not permitted, except that material may be duplicated by you for your research use or educational purposes in electronic or print form. You must obtain permission for any other use. Electronic or print copies may not be offered, whether for sale or otherwise to anyone who is not an authorised user. 


\title{
Disorder and dephasing effects on electron transport through conjugated molecular wires in molecular junctions
}

\author{
Daijiro Nozaki, ${ }^{1}$ Claudia Gomes da Rocha, ${ }^{1,2,{ }^{*}}$ Horacio M. Pastawski, ${ }^{3}$ and Gianaurelio Cuniberti ${ }^{1,4}$ \\ ${ }^{1}$ Institute for Materials Science and Max Bergmann Center of Biomaterials, TU Dresden, 01062 Dresden, Germany \\ ${ }^{2}$ Nanoscience Center, University of Jyväskylä, 40014 Jyväskylä, Finland \\ ${ }^{3}$ Instituto de Fisíca Enrique Gaviola (CONICET) and FaMAF, Universidad Nacional de Córdoba Ciudad Universitaria, \\ 5000 Córdoba, Argentina \\ ${ }^{4}$ Division of IT Convergence Engineering and National Center for Nanomaterials Technology, POSTECH, Pohang 790-784, Republic of Korea
}

(Received 25 October 2011; revised manuscript received 8 February 2012; published 30 April 2012)

\begin{abstract}
Understanding electron transport processes in molecular wires connected between contacts is a central focus in the field of molecular electronics. Especially, the dephasing effect causing tunneling-to-hopping transition has great importance from both applications and fundamental points of view. We analyzed coherent and incoherent electron transmission through conjugated molecular wires by means of density functional tight-binding theory within the D'Amato-Pastawski model. Our approach can study explicitly the structure/transport relationship in molecular junctions in a dephasing environmental condition using only a single dephasing parameter. We investigated the length dependence and the influence of thermal fluctuations on transport and reproduced the well-known tunneling-to-hopping transition. This approach will be a powerful tool for the interpretation of recent conductance measurements of molecular wires.
\end{abstract}

DOI: 10.1103/PhysRevB.85.155327

PACS number(s): 73.63.-b, 72.10.Bg

\section{INTRODUCTION}

The study of electron transport through nanostructures has been a primary interest in the field of molecular electronics in the last few decades. Recently, increasing attention has been paid to the main role played by phase-breaking processes in molecular junctions. ${ }^{1-5}$ The transition from coherent (tunneling) to incoherent (hopping) regimes is one example of such a process that provides helpful information for the fundamental understanding of transport mechanisms and technological applications. Such transitions have commonly been reported in many studies of electron transfer in molecular systems and electron transport in molecular junctions, for instance, selfassembled monolayers, ${ }^{6,7}$ polymers, ${ }^{6-11}$ and macromolecules such as proteins, ${ }^{12}$ DNAs, ${ }^{13}$ and organic semiconductors. ${ }^{8}$

For further development of molecular devices, the intrinsic mechanisms of charge transport including the dephasing effects and influential factors such as thermal fluctuations need to be understood in detail. In order to evaluate the electronic conductance of nanostructures, the Landauer formula is commonly used. However, this method is restricted to address systems within the coherent regime. In principle, this method must be extended before it can be applied to organic semiconductors or disordered polymers where the dephasing effects play an essential role in electron transport. Normally, many molecular aggregates widely used in organic semiconductor devices exhibit high-disorder features. A proper theoretical description of the transport properties of such defective structures has to take into account not only bandlike coherence but also dephasing mechanisms via a hopping representation. ${ }^{14}$

One of the main sources of dephasing processes is the interaction between electronic states and molecular vibrations, referred as electron-phonon $(e-p h)$ coupling. The $e-p h$ interactions can be experimentally inferred from inelastic tunneling spectroscopy or from thermal resonance broadening. The challenge then concentrates in incorporating $e$-ph interactions into the Landauer approach. The current state of the art as followed by several theoretical works involves the use of density functional theory (DFT) within nonequilibrium Green's function formalism where $e$-ph interaction is modeled by selfenergy terms that can be solved self-consistently. ${ }^{15}$ However, such a strategy can be computationally demanding. Such limitations can compromise the understanding of the main role played by dephasing effects on the charge transport properties of molecular devices, leading to the search for less expensive models that attend to more qualitative interpretations. One example of such a model is Büttiker's scattering approach, ${ }^{1,16}$ in which $e-p h$ coupling is replaced by phenomenological voltage probes capable of inducing phase-breaking process into the molecular conductor. Other approaches using reduced density matrix elements where their time evolution can be described by Redfield equation have also been adopted to describe the physics of electron transfer/transport in molecular systems and their crossover trends between coherent and incoherent regimes. ${ }^{17-19}$ Nevertheless, it is highly desired to investigate such a prominent physical phenomenon using a parameter-free (or using as few parameters as possible) first-principles methodology since a more detailed quantitative study can be rendered using such techniques. Such techniques would enable us to investigate the relationship between the conformational change of a realistic molecule and the change of electron transport.

In this manuscript, we analyzed the main role played by dephasing effects in the transport behavior of organic polymer systems using the Hamiltonian version of Büttiker's scattering approach, which is the D' Amato-Pastawski model. ${ }^{20}$ We show that the conductance of the molecules can be dramatically modified due to the dephasing effect depending on the size of the molecules. Important fundamental questions such as the tunneling-to-hopping transition and influence of thermal fluctuation on transport are also addressed in this work. We demonstrate that dephasing has a significant impact in driving 
the transport regime of molecular systems from a quantum mechanical tunneling to Ohmic behavior for longer molecular wires. In addition, we also investigated how the conformational degree of freedom affects the main features of coherent and incoherent transport regimes in such molecular junctions.

\section{THEORETICAL FRAMEWORK}

We calculated the conductance of molecular wires using the Landauer formula written in terms of Green's functions. ${ }^{21}$ Figure 1(a) shows a schematic picture of the studied system which is composed of a two terminal conducting channel connected to a central scattering region. A sequence of local dephasing probes is incorporated to describe incoherent events that affect the transport properties of the system. The electronic structure of the whole molecular system is represented by the general Hamiltonian; $H=H_{\mathrm{L}}+V_{\mathrm{L}}+H_{\mathrm{M}}+V_{\mathrm{R}}+H_{\mathrm{R}}$, where $H_{\mathrm{L} / \mathrm{R}}$ and $H_{\mathrm{M}}$ represent the left/right electrode and central contributions, respectively. $V_{\mathrm{L} / \mathrm{R}}$ defines the coupling between source/drain electrodes and the molecular wire. In this sense, its electronic propagator can be represented by a retarded Green's function defined as $G^{\mathrm{R}}(E)=[(E+i \eta) I-$ $\left.H_{\mathrm{M}}-\Sigma_{\mathrm{L}}-\Sigma_{\mathrm{R}}\right]^{-1}$, where $\Sigma_{\mathrm{L} / \mathrm{R}}$ are the self-energy elements that include the influence of the contacts. The conductance of a molecular channel at low bias and coherent regime is then obtained via Landauer's formula, $G=G_{0} \operatorname{Tr}\left(G^{\mathrm{R}} \Gamma_{\mathrm{L}} G^{\mathrm{A}} \Gamma_{\mathrm{R}}\right)$, where $\operatorname{Tr}$ is the trace operation, $G_{0}$ is the quantum conductance unit $G_{0}=2 e^{2} / h$, and $\Gamma_{\mathrm{L} / \mathrm{R}}$ represents the broadening function given by $\Gamma_{\mathrm{L} / \mathrm{R}}(E)=i\left[\Sigma_{\mathrm{L} / \mathrm{R}}(E)-\Sigma_{\mathrm{L} / \mathrm{R}}^{\dagger}(E)\right]$.

To include dephasing events in the model, the D'AmatoPastawski formalism is adopted. Within this picture, one includes additional self-energy terms that account for the dephasing processes. The new electronic propagator is then defined as $G_{\mathrm{eff}}^{\mathrm{R}}(E)=\left[(E+i \eta) I-H_{\mathrm{M}}-\Sigma_{\mathrm{L}}-\Sigma_{\mathrm{R}}-\right.$

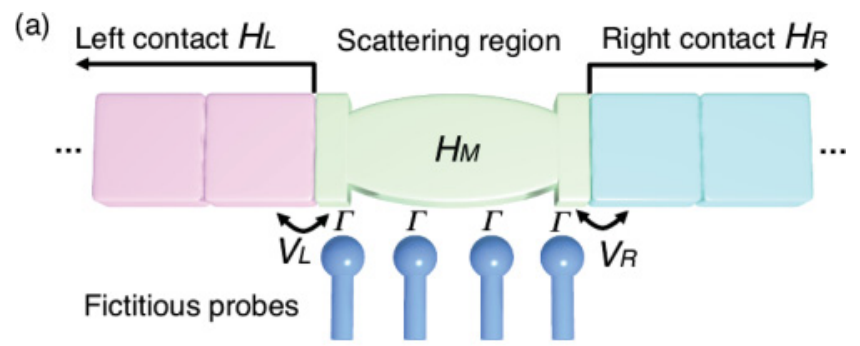

(b)

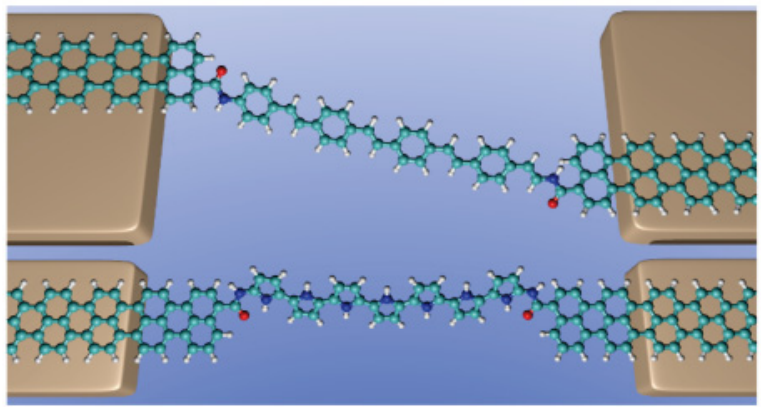

FIG. 1. (Color online) (a) A schematic model of a molecular junction connected between two electrodes. Büttiker probes are introduced to simulate dephasing events. (b) PPV- and (c) pyrroleoligomers are coupled to electrodes consisting of graphene nanoribbons (GNRs) via peptide linkers.
$\left.\sum_{n=1}^{N} \Sigma_{\mathrm{B}, n}\right]^{-1}$, where $N$ is the number of reservoirs and $\Sigma_{\mathrm{B}, n}$ are the self-energies due to Büttiker probes; $\Sigma_{\mathrm{B}, n}=i \gamma_{e-p h} / 2$ that is written in terms of a dephasing parameter, $\gamma_{e-p h}$, associated to the $e$ - $p h$ coupling.

The effective transmission is given by $T_{\text {eff }}=T_{\mathrm{L}, \mathrm{R}}+$ $\sum_{m, n=1}^{N} T_{\mathrm{L}, m} W_{m, n}^{-1} T_{n, \mathrm{R}}$, where $T_{\mathrm{L}, \mathrm{R}}$ describes the coherent contribution and the subsequent term takes into account the incoherent events. $W$ is a Markov matrix formed by transmission elements, $T_{m, n}$ taken between the $m$ th and $n$th probes, and the reflection function $R_{m}=1-\sum_{n \neq m}^{N} T_{m, n}$. At the end, it reads as $W_{m, n}=\delta_{m, n}\left(1-R_{m}\right)-\left(1-\delta_{m, n}\right) T_{m, n}$. The broadening caused by dephasing processes is defined as $\Gamma_{m} \equiv i\left[\Sigma_{\mathrm{B}, m}-\Sigma_{\mathrm{B}, m}^{\dagger}\right]=\gamma_{e-p h}$, which is related to the dephasing rate $k=\Gamma_{m} / \hbar$. Although the dephasing strength $\gamma_{e-p h}$ can be evaluated by the Fermi golden rule quantitatively from first principles, which are comparable to $k_{\mathrm{B}} T, 3,22$ it is also possible to examine the influence of the dephasing events on charge transport by changing the rate of dephasing events.

\section{RESULTS AND DISCUSSION}

The elucidation of our results starts with the electronic transmission curves shown in Fig. 2 obtained within the coherent regime in order to compare it to the incoherent cases. We chose two sample systems for such analysis: PPV- (upper panels) and pyrrole-oligomer (lower panels) polymers. At first, we use a simple tight-binding model, which only considers the nearest-neighboring $\pi$ orbitals to capture the general trends of the transmission profiles. The coupling strength of C-C bonds on the graphene nanoribbon (GNR) electrodes are simply parameterized as $\beta=-2.66 \mathrm{eV}{ }^{23}$ In the scattering region, the transfer integrals for double and single bonds are set as $\beta_{\mathrm{d}}=1.2 \beta$ and $\beta_{\mathrm{s}}=0.8 \beta$, respectively, and the on-site energies for each atom are $\epsilon_{2 p}=0.0(\mathrm{C}), 0.902 \beta(\mathrm{N})$, and $1.902 \beta(\mathrm{O}){ }^{24}$ The Fermi energy $\left(E_{F}\right)$ of the systems are set to 0.0 for simplicity. The coherent transmission results are displayed in Fig. 2 for (a) PPV- and (c) pyrrole-based molecular wires considering different lengths. The transport response of the systems is characterized by a transmission valley centered around the Fermi energy. Such valleys become more pronounced as the molecular length increases resulting in the well-known exponential decay of the conductance at the Fermi level as shown in the logarithm plots of Figs. 2(b) and 2(d) (red lines).

Dephasing effects are subsequently included in the model and we investigate how they affect the conductance behavior as a function of molecular length. Bearing in mind that the $e$-ph coupling is much weaker than the transfer integrals, ${ }^{25}$ we finely changed the dephasing parameters between 0.5 and $20 \mathrm{~m} \beta$ and studied their influence on the charge transport as shown in Figs. 2(b) and 2(d). One can see that the transmission is considerably enhanced in comparison to the coherent tunneling as the length of the molecule increases. A clear deviation from exponential behavior can be observed that becomes more evident with increasing dephasing strength.

More sophisticated methods such as the density functional based tight-binding (DFTB) approach ${ }^{26,27}$ were also employed to verify the robustness of such crossover behavior. We relaxed the molecular junctions and calculated the transmission in the 

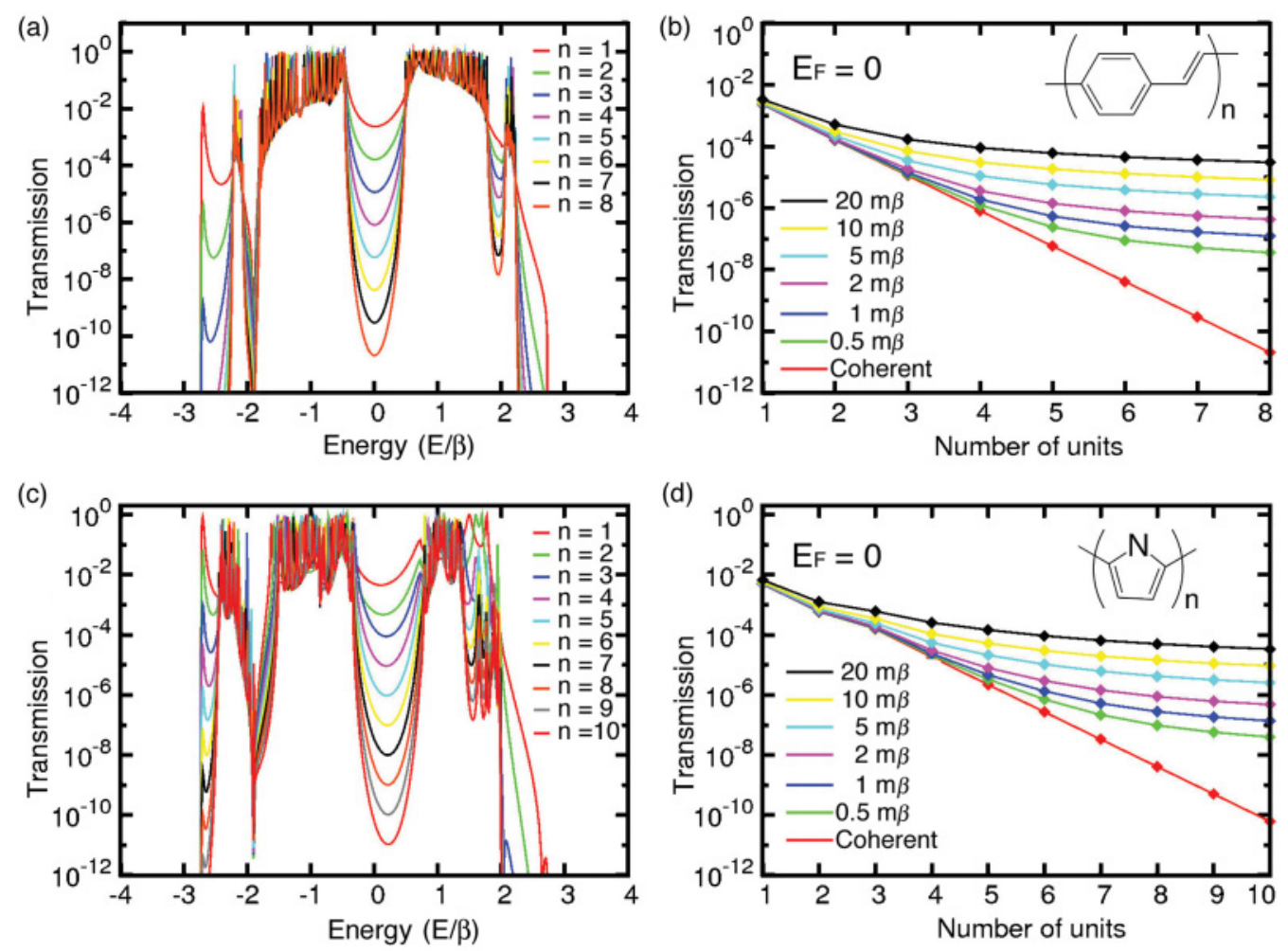

FIG. 2. (Color online) Electronic transmission calculated within tight-binding approximation for (upper panels) PPV- and (lower panels) pyrrole-based molecular wires. Results are depicted as a function of energy [(a) and (c)] at coherent condition and of the molecular length [(b) and (d)] for coherent and incoherent regimes with different dephasing parameters (see legends).

same way as Ref. 28. At first, we relaxed the isolated molecular wires and GNRs individually. Then, we coupled the relaxed isolated molecular wires between the single unit cells of the relaxed GNR electrodes. We performed once more a relaxation procedure on the coupled system using periodic boundary conditions fixing the atoms at the boundaries. Afterwards, the single unit cells of the GNR are transformed into semi-infinite leads, and we calculated the transmission probabilities using the same approach.

The Fermi energy for the system was determined from the electronic structure of an armchair GNR which was found to be $-4.41 \mathrm{eV}$. Because of the steric repulsion between hydrogen atoms, the carbon-carbon distances connecting the unit cells in the horizontal direction are longer than that of others. Therefore the armchair nanoribbon is slightly dimerized giving rise to band gaps around $-5 \mathrm{eV} \cdot{ }^{29}$ As a consequence, an abrupt drop in the transmission functions can be seen in Figs. 3(a) and 3(c). The transmission profiles and length dependence of conductance at the Fermi energy with dephasing effects calculated using DFTB method are also presented in Fig. 3. A similar behavior as the one obtained via simple tightbinding approximation is observed. From the linear trend of coherent tunneling, we determined the damping constants for PPV, $0.241 \AA^{-1}$, and pyrrole, $0.308 \AA^{-1}$. The exponential decay associated with coherent transport is quenched as the dephasing effects get stronger.

Once more, we verified that dephasing dictates the conducting response for longer one-dimensional systems. The change on the transport behavior from direct coherent tunneling to hopping mechanism can be evidently seen in Fig. 4, which shows the resistance as a function of length for both molecular structures calculated with the dephasing strength being $20 \mathrm{meV}$. The system undergoes a crossover from exponential to Ohmic regime with the estimated critical resistance per unit length for PPV and pyrrole being 283.9 and $39.3 \mathrm{M} \Omega / \mathrm{nm}$, respectively. Figure 5 shows the resistance per unit length in the Ohmic regime as a function of dephasing strength for both molecular wires. We can see that the resistance is decreased as dephasing values increase revealing then that incoherent transport is the dominant mechanism.

Since we point at addressing the influence of conformational fluctuations on transport in realistic molecular wires under dephasing conditions, we performed molecular dynamics (MD) simulations at room temperature still using DFTB method and calculated the effective conductance along the $\mathrm{MD}$ trajectories. It is worth mentioning that the MD trajectory should be calculated under nonequilibrium condition and its velocities should include the effect of the heat produced by tunneling current. Nonetheless, for the sake of simplicity, we assumed the system under equilibrium condition. Already under this approximation, it is possible to extract important insights regarding the changes of conductance due to modifications on the atomic structures of the molecules. The motion of the atoms during the simulations has been integrated using the standard velocity Verlet algorithm with a time step of $1.0 \mathrm{fs}$ and a total time duration of $10 \mathrm{ps}$. The influence of structural fluctuations on the conductance is investigated considering that only the atoms in the scattering region are allowed to move. 

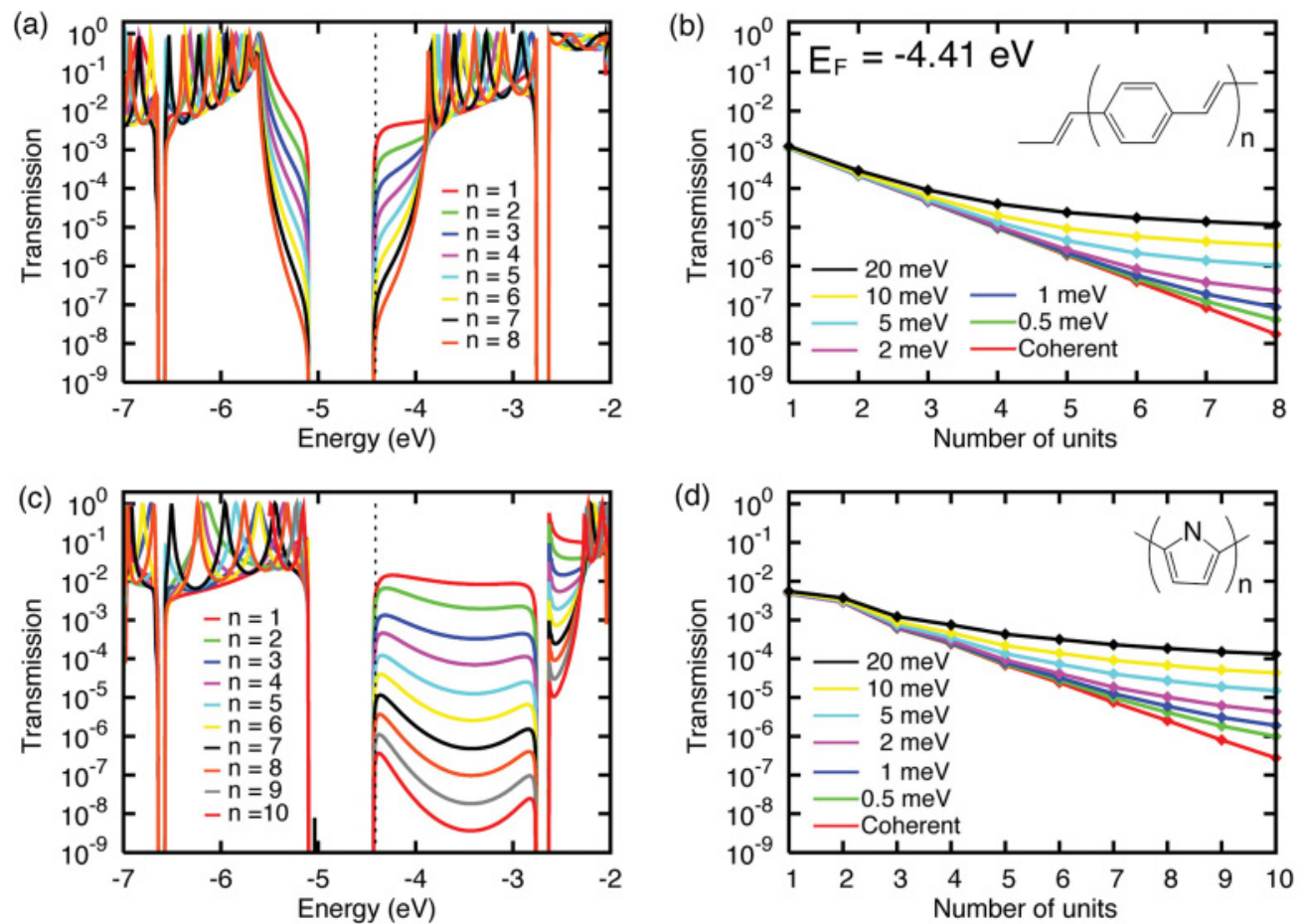

FIG. 3. (Color online) Electronic transmission calculated within DFTB approach for (upper panels) PPV- and (lower panels) pyrrole-based molecular wires. Results are depicted as a function of energy [(a) and (c)] at coherent condition and of the molecular length [(b) and (d)] for coherent and incoherent regimes with different dephasing parameters (see legends).

After the MD simulations, 100 snapshots were extracted from their generated trajectories. Then we calculated the effective conductance for the 100 selected geometries in order to see its changing due to fluctuations in the atomic coordinates and setting the $e$-ph coupling constant at $25.8 \mathrm{meV}^{3}$

Figure 6 shows how the coherent and effective conductances disperse along the MD trajectory with the molecular lengths. The dominance of hopping mechanisms for longer molecules is still preserved along the entire MD trajectory. The dephased transmission clearly deviates from the exponential trend characteristic of coherent tunneling as the guiding lines on the plots reveal. From this result, it is important to distinguish also these two transport regimes by the degree of dispersion of the transmission logarithm $\left(\log _{10} T\right)$ versus molecular length. We expect that strong fluctuations are seen for the length dependence in the coherent tunneling
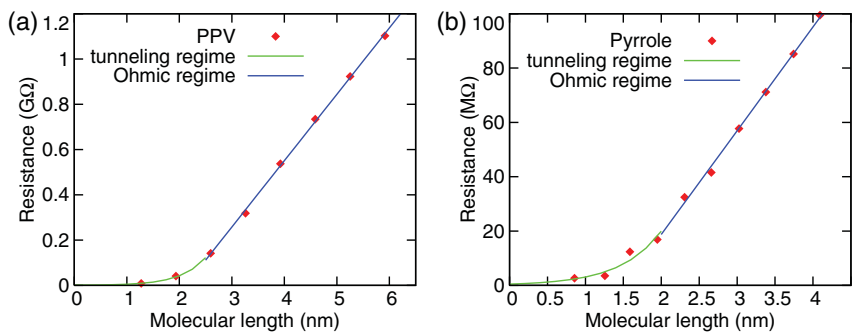

FIG. 4. (Color online) Resistance of (a) PPV- and (b) pyrrolebased oligomers as a function of molecular lengths. The fitting curves for coherent tunneling and hopping transport are shown in green and blue, respectively. Dephasing strength $\gamma_{e-p h}$ was set to $20 \mathrm{meV}$. regime whereas narrower distributions appear when dephasing effects are considered. This can be seen from the variation of the root-mean standard deviation (RMSD) of molecular coordinates with respect to a reference coordinate, in this case, its initial configuration. Figure 7 presents the molecular length dependence of RMSD (left panel) as well as the standard deviation (right panel) of the transmission logarithm $\left(\log _{10} T\right)$ for the coherent and dephased regimes. RMSD results in Fig. 7(a) point out that longer molecules potentially have more configurational degrees of freedom and, for this reason, RMSD increases with the size of the junction. Both types of molecules present approximately the same trends in the RMSD curves.

The standard deviation of $\log _{10} T$ in Fig. 7(b) highlights how strongly the coherent/dephased transport fluctuates along MD trajectory. We can see that coherent tunneling is more
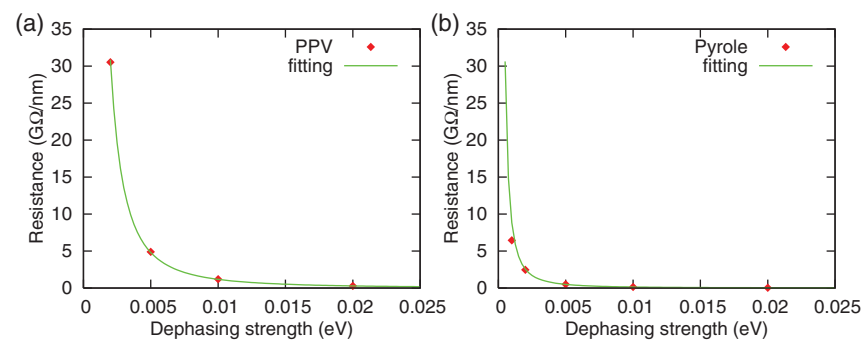

FIG. 5. (Color online) Resistance of (a) PPV- and (b) pyrrolebased oligomers per unit length in the Ohmic regime as a function of dephasing strength. 

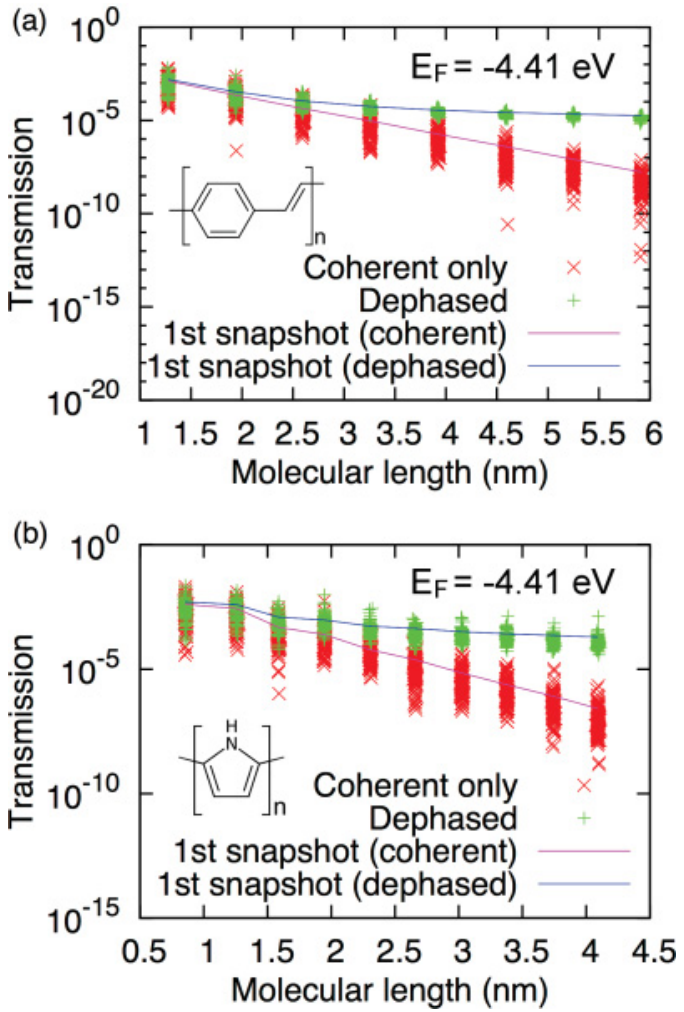

FIG. 6. (Color online) Electronic transmission at the Fermi energy within coherent and incoherent regimes as a function of length on the molecular dynamic flies for PPV-based (upper panel) and pyrrole-based molecular wires (lower panel). In the incoherent regime, dephasing strength was set to $25.8 \mathrm{meV}$.

sensitive to the molecular disorder induced by the thermal fluctuations than dephased transport. Thermal fluctuations can disrupt the $\pi$-orbital conjugation of the molecules, localizing the states along the molecular wires. Such breaking of the delocalized $\pi$ orbitals, which bridge the electron tunneling between two contacts, results in a large dispersion of coherent transmission in the $\log _{10} T$ versus length plots in Fig. 6 . On the other hand, unlike the coherent regime, dephased transport does not fluctuate so strongly with the breaking of $\pi$-orbital delocalization since there are more possibilities for the electrons to propagate through the channel by executing individual jumps with probability $T_{m, n}$ via sequential hopping processes. In other words, there will be always available pathways for hopping transport which will favor the electronic transmission (note that dephased transport is determined by the sum of coherent tunneling and sequential hopping contributions). Therefore dephased transport is less sensitive to thermal fluctuations than coherent tunneling even if the $\pi$-orbital conjugation is thermally disturbed. Another important feature in the $\log _{10} T$ deviation within dephased transport is that it decreases for longer molecules. This is simply caused by the fact that the number of paths through which the charge can be transmitted increases as the molecule extends. The averaging over the increased number of hopping pathways results in a smaller transmission dispersion of dephased transport.
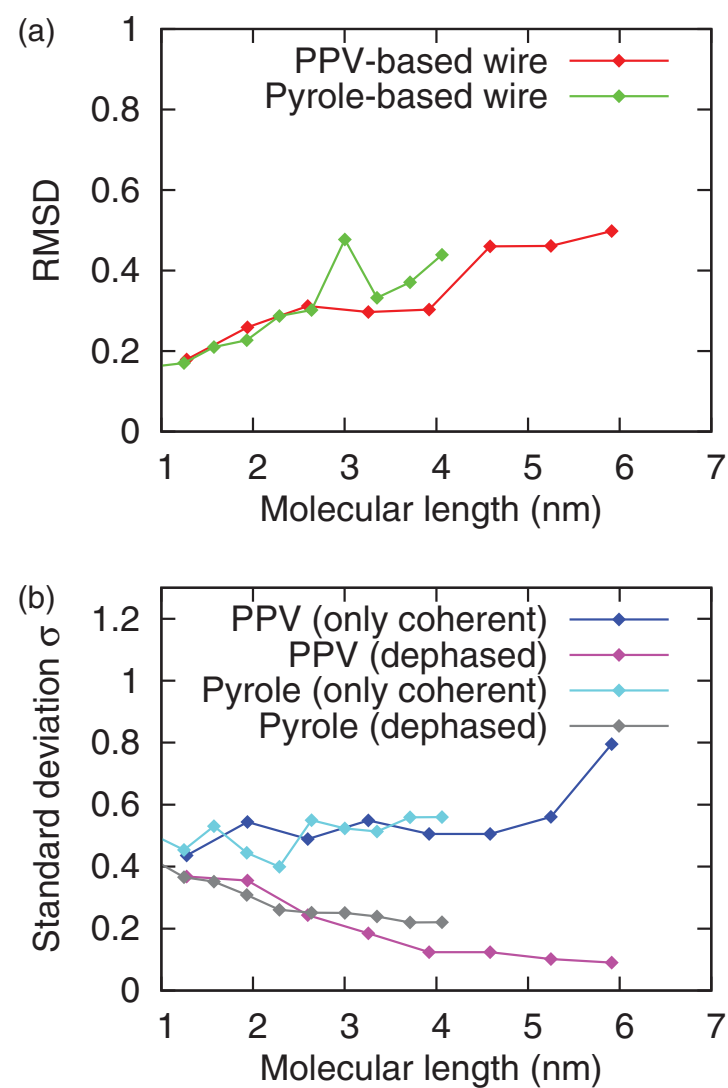

FIG. 7. (Color online) (a) Root-mean standard deviation (RMSD) of two types of molecular wires along MD pathways as a function of molecular lengths. (b) Standard deviation of transmission logarithm $\left(\log _{10} T\right)$ for two types of molecular wires with/without dephasing effects.

\section{CONCLUSION}

In summary, we have investigated electron transport in the presence of dephasing effects by analyzing both the coherent and incoherent process in molecular wires connected between two contacts. By employing the D'Amato-Pastawski model within density-functional based techniques, two transport mechanisms were examined at room temperature where the structural conformations of the systems were also explicitly treated. We were able to observe the tunneling-to-hopping transition-from exponential (coherent) to Ohmic (incoherent) regimes - as the molecular length varies by using only one control parameter. We also investigated the influence of thermal fluctuations and found that the transmission distributions for tunneling transport are broader in comparison to those for the hopping mechanism. This highlights that the coherent component is rather more sensitive to structural disorder than the incoherent one. Such findings reveal that our approach provides a reliable simulation platform for the interpretation of transport measurements performed in molecular systems.

\section{ACKNOWLEDGMENTS}

This work was funded by the Volkswagen Foundation and by the World Class University program through the Korea 
Science and Engineering Foundation funded by the Ministry of Education, Science and Technology (Project No. R31-2008-
000-10100-0), Erasmus Mundus, MPI-PKS, and Alexander von Humboldt Foundation.
*Corresponding author: cgomes@nano.tu-dresden.de

${ }^{1}$ J. Maassen, F. Zahid, and H. Guo, Phys. Rev. B 80, 125423 (2009).

${ }^{2}$ M. Zilly, O. Ujsaghy, and D. E. Wolf, Eur. Phys. J. B 68, 237 (2009).

${ }^{3}$ C. J. Cattena, R. A. Bustos-Marun, and H. M. Pastawski, Phys. Rev. B 82, 144201 (2010).

${ }^{4}$ M. Dey, S. K. Maiti, and S. N. Karmakar, Org. Electron 12, 2017 (2011).

${ }^{5}$ D. Nozaki, Y. Girard, and K. Yoshizawa, J. Phys. Chem. C 112, 17408 (2008).

${ }^{6}$ S. H. Choi, B. Kim, and C. D. Frisbie, Science 320, 1482 (2008).

${ }^{7}$ L. Luo, S. H. Choi, and C. D. Frisbie, Chem. Mater. 23, 631 (2011).

${ }^{8}$ W. B. Davis, W. A. Svec, M. A. Ratner, and M. R. Wasielewski, Nature (London) 396, 60 (1998).

${ }^{9}$ T. Hines, I. Diez-Perez, J. Hihath, H. Liu, Z.-S. Wang, G. Zhou, K. Müllen, and N. Tao, J. Am. Chem. Soc. 132, 11658 (2010).

${ }^{10}$ R. Yamada, H. Kumazawa, S. Tanaka, and H. Tada, Appl. Phys. Express 2, 025002 (2009).

${ }^{11}$ Y. Selzer, M. A. Cabassi, T. S. Mayer, and D. L. Allara, J. Am. Chem. Soc. 126, 4052 (2004).

${ }^{12}$ S. Isied, M. Y. Ogawa, and J. F. Wishart, Chem. Rev. 92, 381 (1992).

${ }^{13}$ C. J. Murphy, M. R. Arkin, Y. Jenkins, N. D. Ghattia, H. Bossmann, N. J. Turro, and J. K. Barton, Science 262, 1025 (1993).

${ }^{14}$ A. Troisi, Chem. Soc. Rev. 40, 2347 (2011).

${ }^{15}$ N. Sergueev, D. Roubtsov, and H. Guo, Phys. Rev. Lett. 95, 146803 (2005).

${ }^{16}$ M. Büttiker, Phys. Rev. Lett. 57, 1761 (1986).
${ }^{17}$ A. K. Felts, W. T. Pollard, and R. A. Friesner, J. Phys. Chem. 99, 2929 (1995).

${ }^{18}$ D. Segal, A. Nitzan, M. Ratner, and W. B. Davis, J. Phys. Chem. B 104, 2790 (2000).

${ }^{19}$ D. Segal, A. Nitzan, W. B. Davis, M. R. Wasielewski, and M. A. Ratner, J. Phys. Chem. B 104, 3817 (2000).

${ }^{20}$ J. L. D'Amato and H. M. Pastawski, Phys. Rev. B 41, 7411 (1990).

${ }^{21}$ S. Datta, Electronic Transport in Mesoscopic Systems (Cambridge University Press, Cambridge, 1995).

${ }^{22}$ H. M. Pastawski, L. E. F. Foa-Torres, and E. Medina, Chem. Phys. 281, 257 (2002).

${ }^{23}$ J. Cai, P. Ruffieux, R. Jaafar, M. Bieri, T. Braun, S. Blankenburg, M. Muoth, A. P. Seitsonen, X. Feng, K. Müllen, and R. Fasel, Nature (London) 4, 470 (2010)

${ }^{24}$ J. G. Verkade, A Pictorial Approach to Molecular Bonding and Vibrations (Springer, Berlin, 1997).

${ }^{25}$ M. Galperin, M. A. Ratner, and A. Nitzan, Nano Lett. 4, 1605 (2004).

${ }^{26} \mathrm{http}: / /$ www.dftb-plus.info and http://www.dftb.org.

${ }^{27}$ M. Elstner, D. Porezag, G. Jungnickel, J. Elsner, M. Haugk, T. Frauenheim, S. Suhai, and G. Seifert, Phys. Rev. B 58, 7260 (1998).

${ }^{28}$ D. Nozaki and G. Cuniberti, Nano Res. 2, 648 (2009).

${ }^{29}$ Y. W. Son, M. L. Cohen, and S. G. Louie, Phys. Rev. Lett. 97, 216803 (2006). 